\title{
A Design of Remote Monitoring System based on 3G and Internet Technology
}

\author{
Shouxian WEN \\ Key Lab of Intelligent Computing and Signal Processing of \\ Ministry of Education, Anhui University \\ Hefei, China \\ Xingguo SUN \\ Key Lab of Intelligent Computing and Signal Processing of \\ Ministry of Education, Anhui University \\ Hefei, China
}

\author{
Lei XU \\ Key Lab of Intelligent Computing and Signal Processing of \\ Ministry of Education, Anhui University \\ Hefei, China \\ Xiaohui LI* \\ Key Lab of Intelligent Computing and Signal Processing of \\ Ministry of Education, Anhui University \\ Hefei, China
}

\begin{abstract}
Aiming at the low-rate and short-distance communication in existing temperature and humidity monitoring system, this paper designs a remote monitoring system based on 3G and Internet technology. This system consists of data acquisition module and data remote transmission module. Atmega128 is used to be as a master control chip of data acquisition module, which realizes the data acquisition and pretreatment, and the collected data is transmitted to the host node by the low-power wireless transmission module NRF905 which is installed on slave nodes, and then the converged data is transmitted to Internet by $3 G$ module. The system can realize remote monitoring, centralized management and so on. The experimental results show that the system has the advantages of low installation cost, low consumption, high sensitivity, high accuracy, long-distance communication and high rate etc.
\end{abstract}

Keywords-DS18B20; 3G; NRF905; Standard Definition (SD) card; Data acquisition subsystem; Data remote transmission subsystem.

\section{INTRODUCTION}

With the rapid development of computer networks and communication technologies, remote monitoring technology in the data acquisition and analysis has been widely used [1-7]. The traditional data acquisition system generally uses the computer (PC) - slave node mode. Slave nodes use a wired connection so that wiring tedious, poor scalability. Especially for the vast space environment, if a wired connection is used, the cost and power consumption is high. In addition, in the traditional wired data transmission system, RS-485 protocol which transmission distance is short is frequently used in the communication between PC and slave nodes. The existing Zigbee protocol can set up remote communication transmission platform through the wireless AD hoc network, but its transmission rate is low. NRF905 wireless module transmission rate is high, but it lacks network protocol [811].The data remote transmission system which composes of $3 \mathrm{G}$ and Internet can achieve a wide range of central control and remote operation. However, if $3 \mathrm{G}$ module is used in each slave node, the cost will be unacceptable.

${ }^{*}$ Corresponding author.

Email address: xhli@ahu.edu.cn (Xiaohui LI).
For these reasons, this paper designs a remote monitoring system based on $3 \mathrm{G}$ and Internet technology. The system consists of data acquisition and data remote transmission subsystem. First, the data acquisition subsystem acquires information, such as temperature, humidity, and then uploads the data to the server that has a fixed IP add ress through remote data transmission subsystem. Through the reasonable design of the application software, the global authorized user can realize the remote data of observation.

\section{SYSTEM FRAMEWORK}

The system framework is shown in Figure 1. The system framework consists of data acquisition subsystem and data remote transmission subsystem. Data acquisition subsystem consists of one host node and several slave nodes. The host and each slave node exchange data by using NRF905 wireless module. The slave node collects information by digital sensors which are inside of the node, and sends it to the host node in form of packets. The converged data via $3 \mathrm{G}$ is connected to Internet, and uploaded to the server. The server is responsible for forwarding the data to the client. Monitoring results will be shown to the users after the client data analysis.

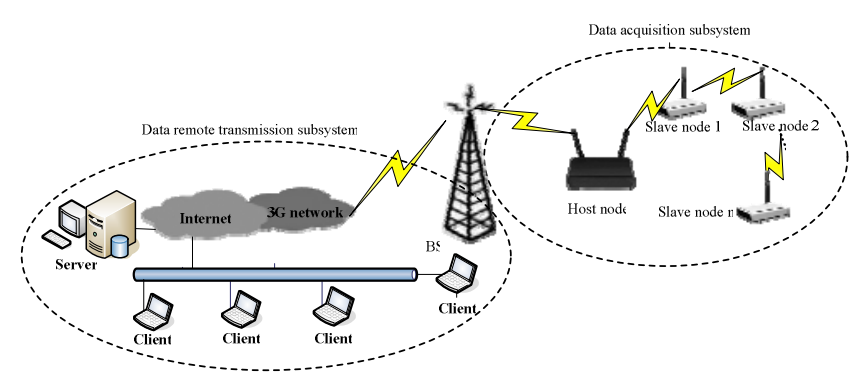

Figure 1. The system framework 


\section{THE DESIGN OF DATA ACQUISITION SUBSYSTEM}

\section{A. The design of hardware circuit}

\section{1) The design of host node hardware circuit}

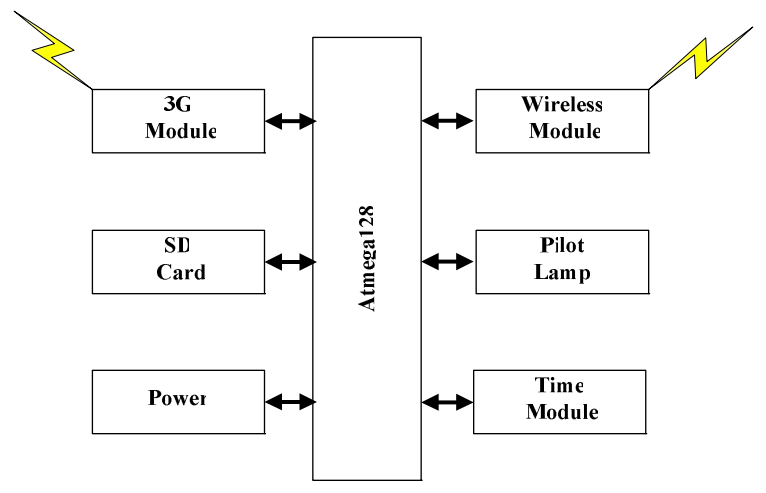

Figure 2. The diagram of host node

As a coordinator for the entire network, the host node is mainly responsible for sending network signal, establishing the network, managing the network node, storing network node information, and finishing the transmission of information.

The diagram of host node is shown in Figure 2. The peripheral circuits of host node includes NRF905 wireless module, $3 \mathrm{G}$ module, SD storage module, indicator module, power module, and the clock module. A 8-bit low-power micro-controller Atmega128 is selected as the processor of the host node. The wireless module adopts NRF905 RF chip, working in the $433 \mathrm{MHz}$ band. It is mainly responsible for wireless networking of the slave node, completing the data transmission of the data acquisition subsystem. 3G module uses the CDMA2000 standard, which is mainly responsible for uploading the aggregate collection information to the server remotely via data transmission subsystem. Via serial peripheral interface (SPI), SD storage and MCU achieve high-speed and accurate data exchange. As the basic unit in SD storage, each sector of 512 bytes stores routing information, network node information, inspection information of temperature and humidity, etc. The indicator module uses a variety of signal lights to reflect the different state of the host node. Because of high instantaneous current of the power supply is needed at the beginning of sending information. LM2576 is used as power module.

\section{2) The design of slave node hardware circuit}

As relays and terminals of the entire network, the slave nodes are primarily responsible for the acquisition and transmission of the data information of the scene temperature, humidity, etc. The diagram of slave node is shown in Figure 3.

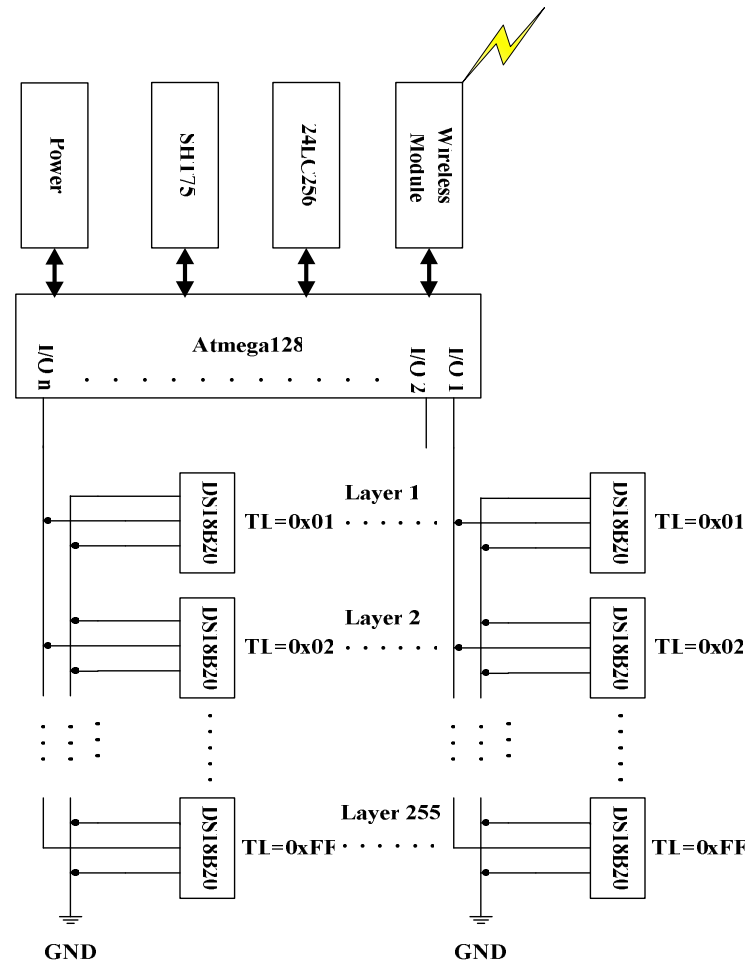

Figure 3. The diagram of slave node

The main composition of the slave node is NRF905 wireless module, data acquisition module, memory module, power module, etc. For slave nodes, Atmega128 is selected as the processor and uses digital sensors DS18B20 to acquire field temperature. Taking the advantages of 1-wire bus, each $\mathrm{I} / \mathrm{O}$ port can be mounted many sensors. Meanwhile, power mode of DS18B20 is parasitic, as shown in Figure 3. In this mode, not only can the power consumption be reduced, but also the slave node can continue to work even if a sensor is short circuit on the bus. With the location information of TH and TL storage sensor in the DS18B20 temperature alarm triggers, the sensor is located. At the time of the slave node power-up initialization, it makes DS18B20 serial number and physical address sequentially stored in the storage module 24LC256. After the slave node receiving instructions, it first reads sensor serial number and physical address from the storage module, then collects temperature information and stores temperature data. Humidity sensor adopts SHT75.

\section{B. The design of software}

1) The design of host node software

The software flow diagram of slave node is shown in Figure 4. 


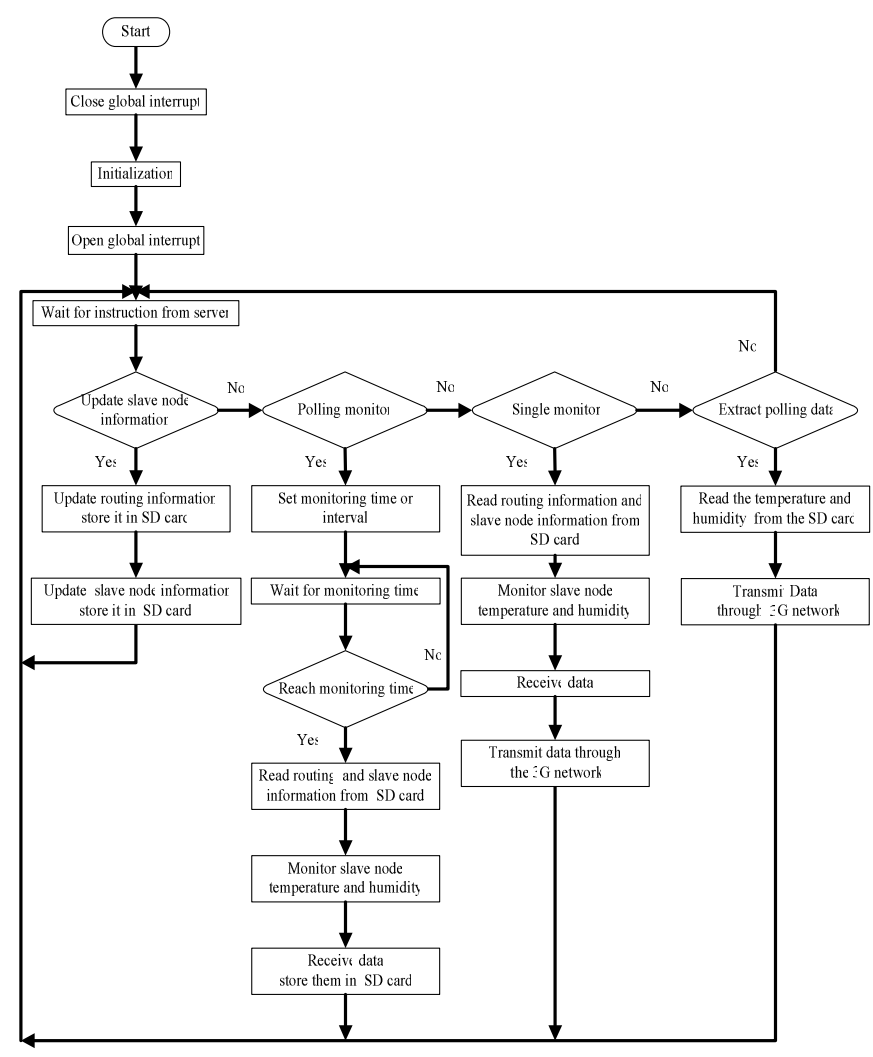

Figure 4. The software flow diagram of host node

When the host node powers on, it first initializes NRF905 wireless module, 3G module, SD storage module, clock module, and register configuration. After initialization, it switches to the interruption mode and waits for instructions from data remote transmission subsystem. The host node will receive the instruction of updating the information of slave nodes before receiving monitoring instructions and finish wireless communication with slave nodes through NRF905 wireless module so that it $\mathrm{c}$ an get the best routing and the distribution information of the slave node sensors and store them in SD storage module of the host node. There are two modes named "polling monitor" and "single monitor". The host node can choose one of them according to the received instructions. Monitor time point and time interval are contained in "polling monitor" instruction and the host node sets monitor time information according to the instruction. The host node firstly reads the configuration information and the routing information of slave nodes from the SD card and then sends instructions to the slave node when the monitor time is up. Finally the host node completes monitoring data convergence of slave nodes, and stores the data in SD card. When the host node receives the instruction of extracting data of polling monitoring mode, it will extract and transmit the data to server by the data remote transmission subsystem. When the host node receives the instruction of single monitoring, the data information will directly be transmitted to the server without being stored in SD card.

TABLE I. DATA FRAME FORMAT

\begin{tabular}{|l|l|l|l|l|l|}
\hline Type & Route & Route_depth & Route_direction & Data & CRC \\
\hline
\end{tabular}

In the transmission subsystem, the NRF905 wireless module data frame format of network communication protocol is shown in table 1.The "Type" indicates the type of command frame and is formed by one byte. "Route" is formed by 5 bytes which indicates routing path, the network number of the host node is " 01 ", and the network number of each slave node is different and unique, which ranks from 02 to 255 . As the host node can't directly communicate with the slave node, it can find the node that it wants to communicate with though the other three slave nodes as relays at most. For instance, "01 02 030405 " indicates the host node will find No.05 slave node through No.02, No.03 and No.04 relays nodes. "Route depth" is defined as routing depth and its value ranks from 01 to 05 . It can determine that which slave node is the next one by combining with routing path. For example, "01 02030405 03" indicates the next node is No. 03 slave node. "Route _direction" is defined as routing direction and its value is " 01 " or " 02 ". " 01 " stands for down-link. " 02 " stands for up-link. The transmitted data is valid while "Route direction" is " 02 ". Data is formed by 11 bytes, and that Data 1 named mark byte indicates valid data bytes and its value is $00 \sim 0 \mathrm{AH}$. Data2 Data11 stores the transmitted data. CRC is used as the check of data frames.

Each transmission module in wireless local area network adopts half duplex communication mode. The slave node in data acquisition subsystem will response and transmit the acquired data to the host node only when receiving the instruction from the data remote transmission system, and the data remote transmission subsystem can send other instructions only after receiving all data. The system will automatically retransmit instructions if the received data is lost or not integrity. If the instruction is send more than 8 times, the system will remind users to check slave nodes.

2) The design of slave node software

The software flow diagram of slave node is shown in Figure 5.

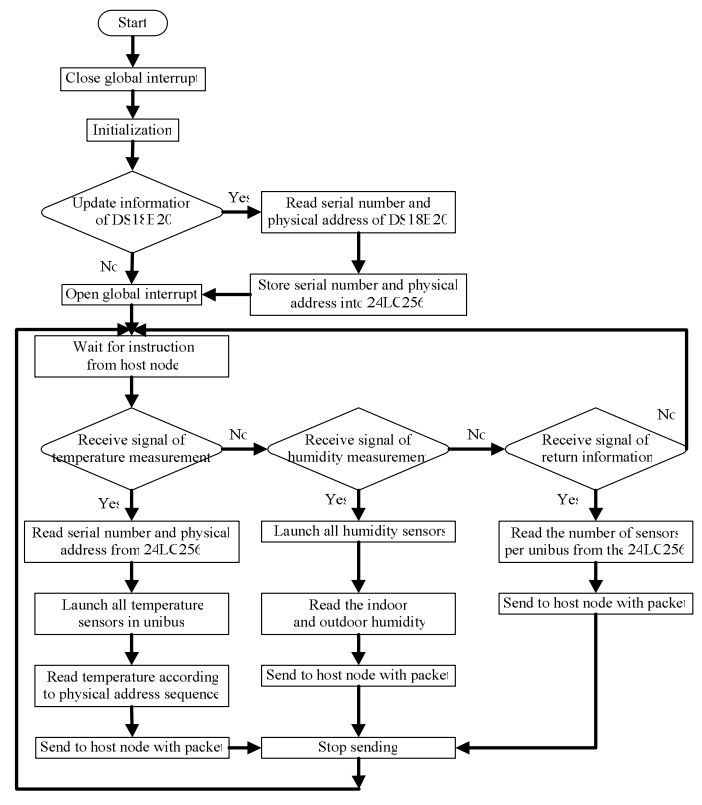

Figure 5. The software flow diagram of slave node 
Once the slave node is powered on, the modules will initialize, including NRF905 wireless module, 24LC256 storage module. Because of the large quantities of DS18B20 in each slave node, long initialization time will be taken. To avoid initialization every time power on, a sign bit is designed in the EEPROM of Atmega128 MCU. Then user can decide whether have the initialization of DS18B20 or not after poweron. During the process of initialization of DS18B20, each bus is pulled up in turn. Then, the serial number and the physical address of each DS18B20 can be read through the binary tree addressing, which are stored in 24LC256. After initialization, interrupt mode will be set, and the slave node will wait for the instruction from the host node. When the slave node receives temperature instruction, the serial number and physical address of DS18B20 will be read from 24LC256. The slave node reads the temperature information according to DS18B20 physical address and sends it to the host node in form of packets. When the slave node re ceives humidity instruction, it reads the outdoor and indoor humidity information and sends it back to the host node. If the instruction that the slave node receives is information instruction, the slave node reads the information of sensors from 24LC256, and sends it back to the host node.

\section{THE DESIGN OF DATA REMOTE TRANSMISSION SUBSYSTEM}

\section{A. Transmission framework}

The data remote transmission subsystem mainly contains client, server and $3 \mathrm{G}$ module. The structure is shown in the Figure 6.

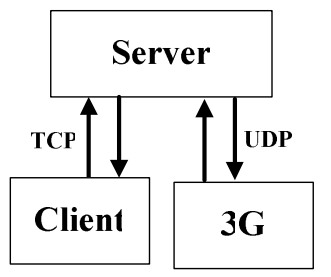

Figure 6. The data remote transmission frame

The user sends instruction to $3 \mathrm{G}$ module through the remote client. According to instruction, the slave node completes the temperature and humidity data collection and controlling. Due to the $3 \mathrm{G}$ module connect to network through the dial-up, it transmits date based on UDP protocol, the IP address and port is ever-changing. To achieve the communication between the client and the $3 \mathrm{G}$ module, there must be a server with fixed IP address as intermediary.

\section{B. The process and experiment results}

The process of data remote transmission subsystem can be divided into two parts, communication between client and server and communication between $3 \mathrm{G}$ module and server.

With reliable data transmission, it is necessary to improve the transmission rate. UDP protocol between $3 \mathrm{G}$ module and server can reduce the system cost, and improve the transmission speed. The instruction retransmission mechanism in client can overcome the trouble that UDP transmission is unreliable. Before $3 \mathrm{G}$ module connects to $\mathrm{t}$ he server, the configuration of the module is necessary, which contains server IP address, port, baud rate, the content and cycle of the heartbeat packets, etc. The different content of heartbeat packets can distinguish different $3 \mathrm{G}$ modules. When the $3 \mathrm{G}$ module powers on, heartbeat packet is sent to server at periodic times.

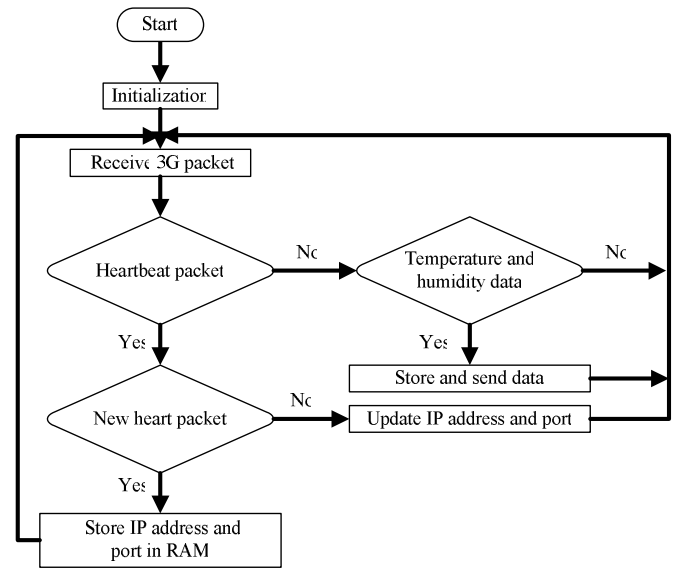

Figure 7. The server work flow diagram (3G module)

The server work flow diagram (3G module) is shown in Figure 7. The server begins to monitor UDP port when it starts. If the server receives new heart packet data, it will store IP address and port number of $3 \mathrm{G}$ module. However, if the heart packet data had been stored, the IP address and port number of $3 \mathrm{G}$ module would be updated. If the server receives temperature and humidity data, it will store data into database according to the information and then send it to the client.

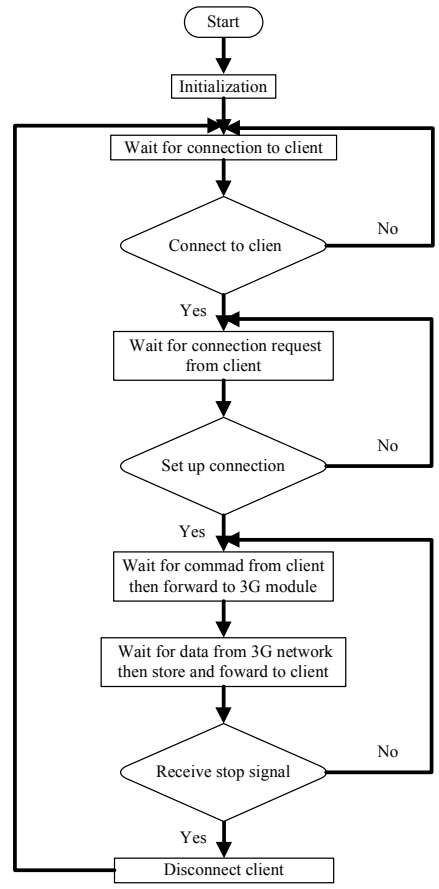

Figure 8. The $\mathrm{C} / \mathrm{S}$ work flow diagram

The $\mathrm{C} / \mathrm{S}$ work flow diagram is shown in Figure 8. When the client connects to the server, it sends a request to the server 
to control 3G module. Each 3G module can only be dominated by one client at the same time. So server response to the client request or not is according to the state of the $3 \mathrm{G}$ module. When $3 \mathrm{G}$ module accepts the request, the client sends instructions to the server by the TCP protocol, and server sends the instructions to $3 \mathrm{G}$ module by the UDP protocol. Then host node collects temperature and humidity data and sends it back to the server by $3 \mathrm{G}$ module. The server stores the data into database, and sends it back to the client. Then the data is displayed on the client.

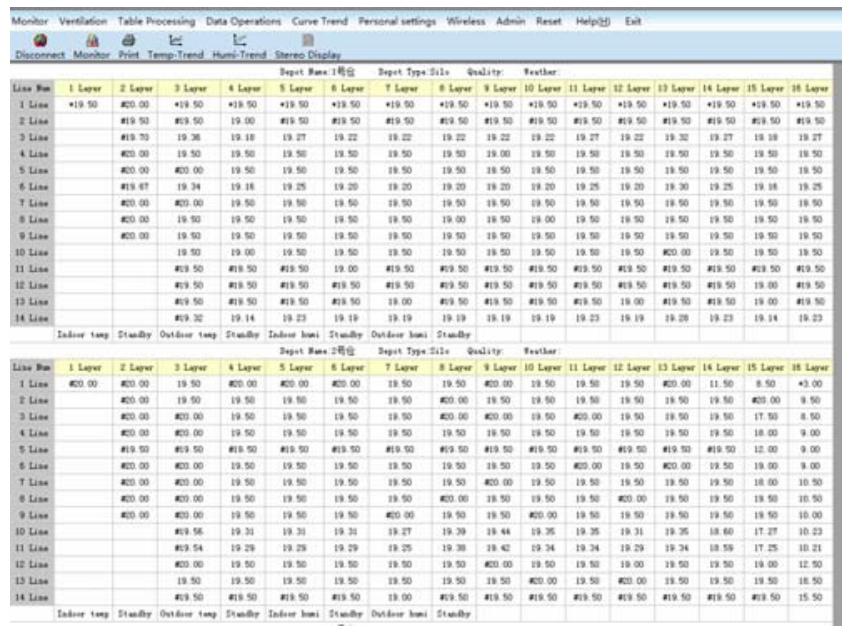

Figure 9. The Measured temperature in server.

The Measured temperature in server is shown in Figure 9. The client software's functions are mainly consisted of realtime data display, historical data view, temperature and humidity data analysis, (such as trend chart of the temperature and humidity data), the perspective display of system temperature and humidity node. By this software, we also can store, view, backup, print, analyze, and display the monitoring data. It provides standardized data interface, which make it simpler to implement the integration with other industrial control software.

\section{CONCLUSION}

In this paper, we use NRF905 wireless RF modules which work in $433 \mathrm{MHz}$ band and $3 \mathrm{G}$ communication module with CDMA2000 standards to design a remote monitoring system. It not only solves the problem of low-rate, short-distance transmission and scene cumbersome wiring, but also reduces high installation cost and power consumption. In addition, it has the advantages of high sensitivity, high accuracy, strong compatibility and efficient operation. Users who have a PC connected to the Internet can get monitoring variety real-time information easily. This system can also be widely used in the fields of industrial and agricultural production, environmental monitoring, traffic monitoring, measurement and control.

\section{ACKNOWLEDGEMENT}

This project is supported by the National Natural Science Foundation of China (No. 60972040), the Anhui Provincial Natural Science Foundation (No. 11040606Q06), the Provincial Project of Natural Science Research for Colleges and Universities of Anhui Province of China (No. KJ2012A003) and the 211 Project of Anhui University.

\section{REFERENCE}

[1] Liu Wei , Zong Xiaoping, "Wireless temperature and humidity collection system design,"heibei.china,vol. 27,2010,pp.500-502.

[2] WangYinling,SunTao, "The Design of Greenhouse Environmental Monitoring of Wireless Sensor Network Nod", Journal of Aricultural Mechanization Reasearch, Vol.33, No.3, 2011

[3] Huang Jianwei. "The wireless temperature measuring system based on DS18B20"henan.china,vol. 29, 2010,pp.118-119.

[4] SUN Xiaodong, "The design of RFID system based on nRF2401[ master degree paper]," Zhejiang: Electrical engineering institute of Zhejiang University, 2008.

[5] Ni Ying, Fu Da-mei, The Design of Greenhouse Monitoring System Based on WSN, Journal of NanJing Institute of Industry Techonology, 2010,10(4)

[6] Yue Pengxia. "Wireless temperature and humidity data acquisition system,"shanxi.china,vol. 2,2009,pp.166-168.

[7] Lu Chao."Wireless temperature and humidity monitor device for Greenhouse,"shanxi.china,vol.7, 2011,pp.136-139.

[8] Wang Li-yan, Yang shi-feng, Design of Greenhouse Environmental Monotoring System Based on Zigbee Technology, Journal of TianJing University of Science\&Technology, 2011,26(1)

[9] XU Lei, ZHANG Hong-wei, LI Xiao-hui*, WU Xian-liang. "A Design of Wireless Temperature and Humidity Monitoring System”, ICCT, pp. 13-16, November 2010.

[10] Qiang Yunxiao,Wu Fengbo."Wireless temperature and humidity measure and control system based on ZigBee technology,"shanxi.china,vol.38,2009,pp.731-734.

[11] YU Haibin, ZEN Peng, "Intelligent wireless sensor network system,"Beijing, Science Press, 2006, pp. 5-6. 